\title{
Effectiveness of acupuncture for breast cancer related lymphedema: protocol for a single-blind, sham-controlled, randomized, multicenter trial
}

Huiru Zhu ${ }^{1 \dagger}$, Jinwan $\mathrm{Li}^{2 \dagger}$, Zheng Peng ${ }^{2 \dagger}$, Yujie Huang ${ }^{2}$, Xiaolan Lv' ${ }^{3}$, Liuying Song ${ }^{2}$, Gechen Zhou², Shengzhang Lin², Jifei Chen², Baoyu He², Fengxian Qin², Xumexiang Liu'², Meiyu Dai ${ }^{2}$, Yan Zou and Shengming $\mathrm{Dai}^{2^{*}}$

\begin{abstract}
Background: Although various treatments for breast cancer related lymphedema exist, there is still a need for a more effective and convenient approach. Pilot studies and our clinical observations suggested that acupuncture may be a potential option. This study aims to verify the effectiveness of acupuncture on BCRL and evaluate its safety using a rigorously designed trial.

Methods/Design: Women who are clinically diagnosed as unilateral BCRL, with a 10\% to 40\% increase in volume compared to the unaffected arm, will be recruited. Following baseline assessment, participants will be randomized to either the real acupuncture group or sham-acupuncture group at a ratio of 1:1, and given a standard real acupuncture or sham-acupuncture treatment accordingly on both arms followed by the same usual care of decongestive therapy. Volume measurements of both arms will be performed for every participant after each treatment. Data collected at baseline and the last session will be used to calculate the primary outcome and secondary outcomes. Other data will be exploited for interim analyses and trial monitoring. The primary outcome is the absolute reduced limb volume ratio. Secondary outcomes are incidence of adverse events and change in quality of life. A t test or non-parameter test will be used to compare the difference between two groups, and assess the overall effectiveness of acupuncture using the SPSS software (version 12).

Discussion: This study will help expand our knowledge about the effectiveness of acupuncture on BCRL, and how acupuncture might be used in the management of this condition. Acupuncture may be a promising complement or alternative to conventional lymphedema treatment methods, if its effectiveness is confirmed.
\end{abstract}

Trial registration: ClinicalTrials.gov NCT02803736 (Registered on October 31, 2016).

Keywords: Acupuncture, Breast cancer related lymphedema, Protocol, Trial

\footnotetext{
* Correspondence: daishm@sina.com

${ }^{\dagger}$ Equal contributors

${ }^{2}$ Department of Clinical Laboratory, the Fourth Affiliated Hospital of Guangxi

Medical University, Liuzhou, Guangxi, China

Full list of author information is available at the end of the article
} 


\section{Background}

As a long-term complication stemming from the disruption of lymph flow, breast cancer related lymphedema (BCRL) is a distressing problem afflicting around 20\% of women who underwent breast cancer treatment, and is characterized by the chronic swelling of one or both upper extremities [1-4]. Depending on the extent of the surgery and auxiliary treatments, BCRL affects patients differently, and may develop one or several years later after surgery [5]. With the increase in breast-cancer incidence as well as the five-year survival rate, it is estimated that there are approximately 530,000 patients facing BCRL in China only [6]. Since patients under this condition often require lifetime management [7], it imposes a big burden on the society with long-term costs, and makes up a significant source of hardship for many families due to health-related productivity loss $[8,9]$. According to research, common risk factors for BCRL includes obesity, axillary lymph node dissection, postoperative radiotherapy, infection, and history of lymphangitis $[10,11]$. Moreover, there is no cure for BRCL so far, and the only way is to avoid risk factors, reduce the severity of symptoms and improve the function of the affected arm. As a result, almost every patient experiencing breast cancer surgery is advised to take precautions and take good care of the affected arm to prevent lymphedema [12, 13]. All these things pose a reminder to patients of their malignant status and become a prominent psychosocial problem that afflicts breast cancer survivors [14, 15]. As a result, the quality of life is much lower for patients after breast cancer surgery than those without this condition [16-19]. At present, there are various treatment options to deal with BCRL, including lymph drainage, low-level laser, micro-surgery, and transplantation of axillary lymph nodes [20-24]. However, no consensus has been reached on any specific approach for BCRL [25], and the management of BCRL remains a major challenge for both physicians and patients [26].

In the theory of Traditional Chinese Medicine (TCM), diseases like BCRL are considered as the same "zheng" as "edema" [27], and is thought to be caused by qi deficiency and blood stagnation. Acupuncture, as an ancient therapy in TCM, is thought to have the efficacy to stimulate the transformation of $\mathrm{qi}$ and drainage of dampness [28], and is widely used for various diseases including edema in East Asia for thousands of years [29]. Previous researches in patients with lymphedema suggest that this treatment might reduce swelling by improving circulation without inducing safety issues [30-35]. However, results regarding its effectiveness in the reduction of swelling have not yet been well established, and no randomized controlled trial has been performed on this issue. Therefore, with this protocol, we aim to evaluate the effectiveness of acupuncture for the treatment of BCRL.

\section{Method \\ Study setting/design}

This multi-center two-arm randomized clinical trial will be performed in the following three medical centers: the Fourth Affiliated Hospital of Guangxi Medical University (about 400 breast cancer patients seen annually), the Third Affiliated Hospital of Guangxi University of Chinese Medicine (about 200 breast cancer patients seen annually), and Liuzhou Maternity and Child Health Care Hospital (about 120 breast cancer patients seen annually). A fixed quota (number of participants recruited) will be distributed to each center based on their total number of breast cancer patients yearly (Additional file 1). The recruitment would start from December, 2016, and will end in October, 2019 synchronously in the three centers. For patients assigned to the real acupuncture group, a standard acupuncture procedure using the real will be performed; for patients assigned to the sham acupuncture group, a standard acupuncture procedure using the sham needles will be performed. The acupoints, frequency (3 times a week for 4 weeks), and duration (30 min after de qi) of each patient in both groups should be the same. All participants in both groups will receive the same usual care of decongestive therapy following acupuncture. An overview of the enrollment procedures, the study design, and the assessments of this trial is illustrated in Table 1.

This study has been approved by the Institutional Review Board of the Fourth Affiliated Hospital of Guangxi Medical University (Approval Number: PJK2016090).

\section{Study oversight}

Three committees will be in charge of the supervision in this trial.

\section{The trial steering committee (TSC).}

The TSC, composed of trial designers of the trial and coordinators of each center, is responsible for key technical and operational problems, such as changes in eligibility criteria, outcomes, analyses, etc. A meeting will be held every month.

\section{The trial coordinating committee (TCC)}

The TCC, composed of coordinators, acupuncturists and nurses in each center, is in charge of specific business, such as recruitment and identification of possible participants, information dissemination of this trial, collection and preservation of data and reports, and management of trial sites. The coordinators in each center 
Table 1 Procedures and time-points of enrollment, intervention, and assessment in this trial

\begin{tabular}{|c|c|c|c|c|c|c|c|c|c|c|c|c|c|c|c|c|}
\hline \multirow{4}{*}{$\begin{array}{l}\text { Time Point } \\
\text { Enrollment: }\end{array}$} & \multicolumn{15}{|c|}{ Study Period } & \multirow{3}{*}{ Close-out } \\
\hline & \multirow{3}{*}{$\begin{array}{c}\text { Identification } \\
- \\
\end{array}$} & \multirow{3}{*}{$\begin{array}{c}\text { Enrollment } \\
- \\
\end{array}$} & \multirow{3}{*}{$\begin{array}{c}\text { Allocation } \\
0 \\
\end{array}$} & \multicolumn{12}{|c|}{ Post-allocation } & \\
\hline & & & & \multicolumn{3}{|c|}{ Week 1} & \multicolumn{3}{|c|}{ Week 2} & \multicolumn{3}{|c|}{ Week 1} & \multicolumn{3}{|c|}{ Week 1} & \\
\hline & & & & & & & & & & & & & & & & \\
\hline \multirow{7}{*}{$\begin{array}{l}\text { Inclusion Criteria } \\
\text { Physical examination } \\
\text { Baseline Assessment } \\
\text { Eligibility Screen } \\
\text { Informed Consent } \\
\text { Unique Sequence No. } \\
\text { Case Report Forms }\end{array}$} & $\mathrm{X}$ & & & & & & & & & & & & & & & \\
\hline & $X$ & & & & & & & & & & & & & & & \\
\hline & $X$ & & & & & & & & & & & & & & & \\
\hline & & $X$ & & & & & & & & & & & & & & \\
\hline & & $X$ & & & & & & & & & & & & & & \\
\hline & & $\mathrm{X}$ & & & & & & & & & & & & & & \\
\hline & & $\mathrm{X}$ & & & & & & & & & & & & & & $\mathrm{X}$ \\
\hline Allocation & & & $\mathrm{X}$ & & & & & & & & & & & & & \\
\hline \multicolumn{17}{|l|}{ Intervention: } \\
\hline \multirow{3}{*}{$\begin{array}{l}\text { Real Acupuncture } \\
\text { Sham Acupuncture } \\
\text { Decongestive Therapy }\end{array}$} & & & & $X$ & $X$ & $X$ & $X$ & $X$ & $X$ & $X$ & $\mathrm{X}$ & $\mathrm{X}$ & $\mathrm{X}$ & $X$ & $\mathrm{X}$ & \\
\hline & & & & $\mathrm{X}$ & $\mathrm{X}$ & $\mathrm{X}$ & $X$ & $\mathrm{X}$ & $\mathrm{X}$ & $\mathrm{X}$ & $\mathrm{X}$ & $\mathrm{X}$ & $\mathrm{X}$ & $\mathrm{X}$ & $X$ & \\
\hline & & & & $\mathrm{X}$ & $\mathrm{X}$ & $\mathrm{X}$ & $X$ & $\mathrm{X}$ & $\mathrm{X}$ & $\mathrm{X}$ & $\mathrm{X}$ & $\mathrm{X}$ & $\mathrm{X}$ & $\mathrm{X}$ & $\mathrm{X}$ & \\
\hline \multicolumn{17}{|l|}{ Assessments: } \\
\hline \multirow{8}{*}{$\begin{array}{l}\text { Perometer } \\
\text { Adverse Events } \\
\text { SRQLQ } \\
\text { Blinding Questionnaire } \\
\text { Blood pressure } \\
\text { Pulse Rate } \\
\text { Blood Sample Test } \\
\text { ECG test }\end{array}$} & $X$ & & & & & $\mathrm{X}$ & & & $\mathrm{X}$ & & & $\mathrm{X}$ & & & $\mathrm{X}$ & \\
\hline & & & & $\longleftarrow$ & & & & & & & & & & & $\rightarrow$ & \\
\hline & & $X$ & & & & & & & & & & & & & & $X$ \\
\hline & & & & $\mathrm{X}$ & & & & & & & & & & & & $X$ \\
\hline & $X$ & & & $\mathrm{X}$ & $X$ & $X$ & $X$ & $\mathrm{X}$ & $\mathrm{X}$ & $\mathrm{X}$ & $\mathrm{X}$ & $\mathrm{X}$ & $X$ & $\mathrm{X}$ & $\mathrm{X}$ & \\
\hline & $X$ & & & $\mathrm{X}$ & $\mathrm{X}$ & $\mathrm{X}$ & $\mathrm{X}$ & $\mathrm{X}$ & $\mathrm{X}$ & $\mathrm{X}$ & $X$ & $X$ & $\mathrm{X}$ & $X$ & $X$ & \\
\hline & $X$ & & & & & & & & $\mathrm{X}$ & & & & & & $X$ & \\
\hline & $X$ & & & & & & & & $\mathrm{X}$ & & & & & & $X$ & \\
\hline
\end{tabular}

are responsible for the establishment of the standard operation procedures and the training of relevant staff prior to the initiation of this trial. The TCC will cooperate with TSC and make a report every month.

\section{The data and safety monitoring board (DSMB)}

The DSMB, composed of experienced experts in experimental design and statistics, is responsible for review and evaluation of the trial periodically. They mainly focus on issues such as execution of the trial, collection of the data, participants' safety, and personal privacy protection. They will also report to the TSC monthly and may provide advice in the modification or termination of the trial.

\section{Eligibility criteria}

In this trial, following inclusion criteria will be adopted:

- women who have completed breast cancer treatment

for more than 1 year;

- no evidence of recurrence;

- unilateral lymphedema resulting from surgery for

breast cancer;

- age between 20 and 45;
- BMI between 18 and 25;

- mild to moderate lymphedema (10\% to $40 \%$ increase in volume compared to the unaffected arm).

Patients will be excluded as follows:

- bilateral lymphedema;

- current use of chemotherapy or radiotherapy;

- history of bilateral axillary lymph node dissection;

- serious lymphedema, $>40 \%$ increase in volume compared to the unaffected arm;

- pregnant women or overweight women;

- unable to adhere to the protocol or the treatment schedule;

- recurrent breast cancer or evidence of other active cancer;

- current use of diuretics like Diosmin, or other investigational drugs;

- contraindications for acupuncture, such as infections, open wounds, or broken skin.

\section{Forbidden drugs and concomitant treatments}

To avoid cross effect, following drugs are unacceptable in this trial, including Diosmin, Hesperidin, and diuretics. Other concomitant treatments should be consulted with the physician in charge and meticulously recorded. 


\section{Recruitment of participants}

The nurses in each center will identify possible participants according to the inclusion and exclusion criteria. Then possible participants will be invited to attend a screening visit, during which a routine physical examination and a baseline assessment including the upper limbs and risks of recurrence will be performed. The coordinators in charge will confirm their eligibility based on their health status and inclusion criteria. For every eligible participant, a unique number will be given by the coordinators based on their enrollment sequence after a written informed consent was obtained, and data on demographic information will be collected through interviews and questionnaires by the acupuncturists funded by this project.

\section{Randomization and allocation}

Eligible participants in each center are randomized into the real acupuncture group and the sham-acupuncture group at a ratio of 1:1 according to the randomization sequence, which is a series of randomization numbers representing each patient's unique number, and pregenerated by the chief designer using RAND function in Microsoft Excel program according to the quota of each center (Additional file 1).

\section{Blinding}

In this trial, participants and statisticians are blinded to the group assignment, whereas acupuncturists are not, due to the nature of this trial. However, to minimize the unintentional physical cues and bias in this trial, acupuncturists will be required to emulate the same procedure for the shamacupuncture groups. And the credibility of blinding will be assessed by experiments before the formal trial, and adjustments will be made until it is acceptable. The process of the blinding will be monitored and assessed by the DSMB.

\section{Intervention}

Eligible participants will be required to set up an appointment by the acupuncturist according to their symptoms patterns, such as the time period their lymphedema manifest the most typical symptoms. Upon arrival at scheduled appointments, participants will receive real or sham acupuncture accordingly on both arms. In this trial, we apply sterilized stainless steel needles $(0.25 \times 40 \mathrm{~mm}$; Asia Med, Munich, Germany) in real acupuncture group, and blunt telescopic placebo needles (Streitberger needle; Asia Med, Munich, Germany) in sham-controlled group. All procedures will be performed by officially registered acupuncturists with clinical experience of 3 years at least.

\section{Real acupuncture group}

Patients in the real acupuncture group will receive true acupuncture 3 times a week for 4 weeks (12 sessions) at
6 acupoints in each arm: Jianyu (LI 15), Jianliao (SJ 14), Binao (LI 14), Quchi (LI 11), Shousanli (LI 10), and Waiguan (SJ 5). These acupuncture points were selected on the basis of classic TCM record, the published literature [30-35], and the consensus between our experienced acupuncturists. Acupoints are detailed in Fig. 1. After the application of alcohol swabs, these acupoints will be covered by adhesive plaster with plastic rings, and stimulated by manual rotation of the needles accompanied by lifting and thrusting gently to make patients get de qi, which is a sensation of aching or distension or numbness elicited by acupoint stimulation $[36,37]$. Then needles will stay put for $30 \mathrm{~min}$.

\section{Sham-acupuncture group}

Patients in the sham-acupuncture group will receive the same sessions of treatment using Streitberger needles at the same acupoints. Streitberger needles have the appearance of the real needles, however, they will not penetrate the skin. They can be placed at the same acupoints as the real acupuncture group, and held in position by adhesive plaster with a plastic ring, which is conducive to the blinding purpose [38]. When the needle is pushed against the skin, a pricking sensation just like the real insertion will be generated. However, as the pressure increases, the needle shaft will bounce back into the handle, which guarantees the skin unbroken and makes an artificial impression of needle insertion (Fig. 2). Other procedures will be the same with the real acupuncture group, including the application of adhesive plaster and plastic rings.

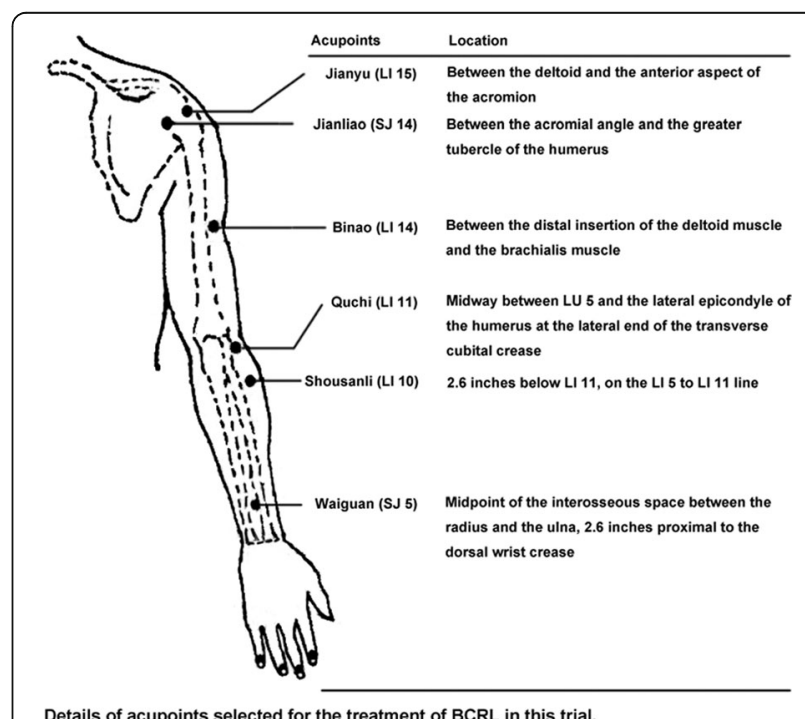

Details of acupoints selected for the treatment of $B C R L$ in this trial.

Fig. 1 Overview of selected acupoints in this trial. Participants will receive real acupuncture or sham-acupuncture at the same acupoints in both arms 3 times a week for 4 weeks (12 sessions) 


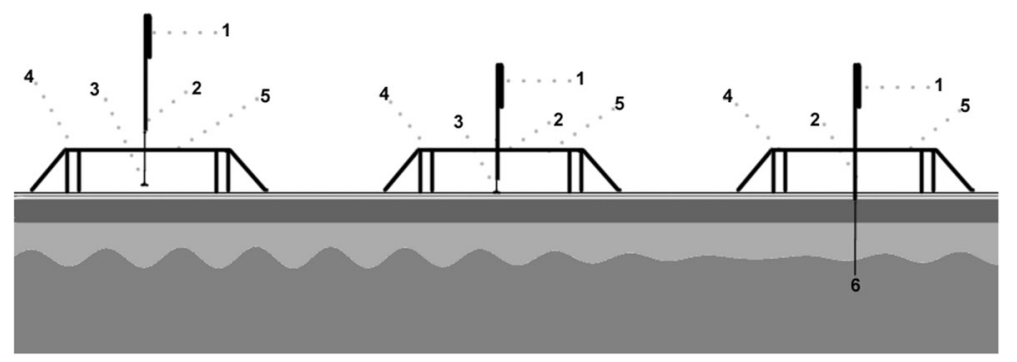

Fig. 2 Structure of Streitberger needle. (1) Needle handle (2) Needle rod (3) Blunt tip of the placebo needle (4) Plastic ring (5) Plastic cover (6) Sharp tip of the real needle

\section{Decongestive therapy}

For ethical considerations (the interest of participants in sham-group), every participant in both groups will receive the same usual care of decongestive therapy after treatment, which includes a daily manual lymph drainage, gentle exercise, applying moisturizer, and wearing elastic compression garments. Table 2 details the duration, frequency, executor, and location of each step. For participants who take other practice, detailed reports will be required.

\section{Patient health monitoring}

For each participant in both groups, blood pressure and pulse rate will be monitored following every treatment session, whereas routine blood test, ECG test, renal function test and liver function test will be conducted every 2 weeks (Table 1). These data will be monitored closely by DSMB during the trial.

Moreover, as cuts and other breakage of the skin would incur infection and the deterioration of lymphedema, all participants will be warned. For those developing sudden onset of infection, the treatment should be discontinued till the patients have fully recovered and the coordinator in charge should be contacted as soon as possible. In other cases like serious complications and occurrence of other emergencies, where patients are unfit for further therapies, they will be persuaded to quit.

\section{Data collection}

Before the first treatment session, every participant will get an assessment of both limbs by perometer, which is an automated infrared light digital scanner equipped with a microcomputer. When the limb is placed inside, the infrared light transmitters located on two sides of the perometer will be activated, and the blockage of the transmission of infrared light from both sides caused by the limb will be recorded and calculated. As the transmitters move along the limb, a series of images will be recorded. Hence a highly accurate measurement of the limb size and volume will be created. And these results will serve as the baseline data. Following the treatment of each session, participants in both groups will be asked to take assessments to get the interim data. Upon the last session, the assessments of both limbs will be adopted as the end-point data. For each arm, the measurement will repeat 3 times to ensure the reliability. Table 1 illustrates all the time points when measurements are taken during the whole procedure.

\section{Primary outcome}

\section{Absolute reduced limb volume ratio (ARLVR)}

The ARLVR is the proportion of absolute reduced limb volume in the affected arm after the therapy, compared with the absolute excess volume in the affected arm before the therapy. This index is inspired by the methods established by Anderson et al. [39] With all data collected at the end of the trial, the ARLVR will be calculated using the following formula:

$$
\operatorname{ARLVR}(\%)=\frac{\left(\mathrm{V}_{\mathrm{AB}}-\mathrm{V}_{\mathrm{AL}}\right)-\left(\mathrm{V}_{\mathrm{UB}}-\mathrm{V}_{\mathrm{UL}}\right)}{\left(\mathrm{V}_{\mathrm{AB}}-\mathrm{V}_{\mathrm{AL}}\right)} \times 100 \%
$$

In particular, $\mathrm{V}_{\mathrm{AB}}$ represents the volume of affected arm with lymphedema at baseline; $\mathrm{V}_{\mathrm{AL}}$ represents the volume of affected arm with lymphedema at the last session; $\mathrm{V}_{\mathrm{UB}}$ represents the volume of unaffected arm at

Table 2 Overview of standard decongestive therapy contents

\begin{tabular}{|c|c|c|c|c|c|}
\hline Standard decongestive therapy & Duration & Frequency & Location & Record & Note \\
\hline Manual Lymph Drainage & $30 \mathrm{~min}$ & Daily & Hospital & Massage Therapist & Meticulous records are needed otherwise. \\
\hline Applying Moisturizer & 3 times & Daily & Home & Participant & \\
\hline Gentle Exercise & $2 \mathrm{~h}$ & Daily & Home & Participant & \\
\hline Wearing Compression Garment & $3 \mathrm{~h}$ & Daily & Home & Participant & \\
\hline
\end{tabular}


baseline; $\mathrm{V}_{\mathrm{UL}}$ represents the volume of unaffected arm at the last session.

\section{Secondary outcomes Incidence of adverse events (IAE)}

The IAE will include every adverse event during the treatment, unless it is confirmed otherwise. Possible adverse events include infection and exacerbation of lymphedema. Participants will be asked to report any significant changes to their health state following treatment. Once adverse event occurs, it should be closely monitored and meticulously recorded until stabilization or resolution, and the chief designer and the coordinators in charge will be informed immediately. Together with the acupuncturist in charge, they will consult on the case, take proper actions and evaluate the severity. Unless it has been confirmed irrelevant with the intervention of this trial, every possible case should be reported timely to the Institutional Review Board. The chief designer is responsible for the report of all adverse events. The DSMB will inspect periodically to ensure that all adverse events are handled properly.

\section{Quality of life}

Quality of life is assessed using a questionnaire from Patient-Reported Outcomes Measurement Information System (PROMIS; http://www.healthmeasures.net/index.php), which requires participants to evaluate their overall health through 10 self-reported global health items representing physical health, pain, fatigue, mental health and social health. Global Physical Health (GPH) and Global Mental Health $(\mathrm{GMH})$ scales will be computed accordingly, and higher scores reflect better life quality. This scoring system has demonstrated remarkable internal consistency and reliability [40].

\section{Sample size calculation}

As no two-arm, randomized, multi-centered, shamcontrolled trial has been performed on this topic, no existing data regarding the sample size are available. However, based on our preliminary clinical data collected and a similar study [41], we assume that the mean and SD of ARLVR in the real-acupuncture group and shamacupuncture group are $(0.32,0.25)$ and $(0.22,0.2)$, respectively, and after calculation using G-Power (V.3.1), the effect size (ES) is 0.4417261 . Next we input the parameters above, set the alpha risk ( $\alpha$, type 1 error risk) and beta risk ( $\beta$, type II error risk) at 0.05 and 0.2 respectively, and choose "t test from two independent groups" and "A Priori: Compute required sample size given $\alpha$, power and effect size", then after calculating, the required sample size is 164 (Additional file 2). Assuming the loss to follow-up rate is $20 \%$, then 197 is the total minimum sample size required in this trial. However, to increase the power and make full preparation, we decided to recruit 200 participants, 100 for each arm in this trial.

\section{Loss to follow-up and data management}

In practice, however, participants might withdraw from the treatment program at any time. In that case, a call from the acupuncturist in charge will be given to the participant, and appropriate advice and assistance will be provided. If a participant insists on withdrawing from this trial, an instant evaluation of lymphedema status will be made, if it is possible. Complete case reports and case reports on loss-to-follow-up of the participants will be reported to the coordinators in charge every month. First-hand data will be recorded by the nurse and the acupuncturist. The coordinator in each center will collect and preserve the data every two weeks. When the trial is done, the aggregated data will be extracted by two independent researchers to guarantee the accuracy, and finally submitted to the principal investigator for analysis. During the whole process, the privacy of research participants will be fully protected.

\section{Statistical and data analysis}

Upon the receipt of the final data, the KolmogorovSmirnov test (K-S test) will be performed, followed by a test for homogeneity of variance to see whether the variances are equal between groups. If the data distribution fits a normal distribution with equal variance, the independent $t$ test will be employed; otherwise, nonparametric test will be chosen to determine whether there is significant difference between the real acupuncture group and the sham acupuncture group. Moreover, one-way ANOVA will be performed to see whether there is significant difference between three centers. Analysis of covariance adjusted for clinical center and baseline will be applied on the condition that significant difference exists between three centers. In addition, regression analysis will be performed to analyze the effects of co-variances (risk factors) such as BMI, axillary lymph node dissection, postoperative radiotherapy, infection, and history of lymphangitis on the overall outcome of this treatment. A type I level error $<0.05$ will be interpreted as statistically significant and $95 \%$ CI, mean, and SD will be calculated. Data analysis in this trial will consist of an intent-to-treat (ITT) analysis, a per-protocol (PP) analysis and an interim analysis. The ITT analysis will collect data from all the participants in this trial, and for those loss to follow-up, the last observation carried forward method will be implemented. As for the PP analysis, data collection will be rigorous, and only those participants who strictly comply with the protocol are eligible. The interim analysis in this trial performed by DSMB, will use the interim data collected during the trial to actively monitor the operating status of the 
trial and help find problems timely. All statistical analyses will be conducted using the SPSS software (version 12).

\section{Discussion}

BCRL can impose great burden on the society and cause significant physical and psychological morbidity to patients [42]. Despite the wide application of less invasive surgical techniques, risk for the development of BCRL still remains [43]. Current treatments options for BCRL are either expensive or rather time consuming [44]. Traditional acupuncture emerges as an efficacious, economical, and convenient choice to manage this chronic condition with few side effects, according to several pilot reports [30-35] and our clinical observation. However, after a thorough search in Pubmed and Embase for relevant articles, we found existing studies on this issue were case reports, small single-arm studies [30-33], or parallel studies using regular routine management or Diosmin as a comparison [34, 35], which cannot rule out the placebo effect and needs to be confirmed by rigorouslydesigned, large-scale studies. The efficacy of real acupuncture over sham acupuncture in dealing BCRL has not been fully illustrated. Therefore, additional research is required to confirm the efficacy and cost-effectiveness of acupuncture on BCRL.In this study, we will adopt Streitberger needle as the sham-acupuncture instrument. Compared with other methods such as placebo points acupuncture, shallow acupuncture at real acupoints and shallow acupuncture at placebo points, Streitberger needle have significant advantages, which make it a perfect choice to keep consistency and credibility, and avoid possible efficacy brought by placebo point acupuncture or shallow acupuncture. This method has been used in several studies with good performance, and was recommended as a reliable sham control [45-47]. Another merit of this study is the measurement instrument we choose. As pointed out above, perometer can easily and swiftly measure the volume of a participants' limb with high reliability and minimal inter-operator variability compared with other conventional methods, such as volumeters (using a water displacement tank to measure volume) and tape measures (using a tape to measure limb circumference) [48-50], although the measurements from different methods are not interchangeable [51]. According to the data collected from patients and previous reports, the day to day variability in limb volumes is around 0.5 [52]. What is more, to better embody the real effect of acupuncture on BCRL, we use the ARLVR, a more reasonable and practical index that is calculated based on the surplus between the affected arm and the contralateral arm. Theoretically, it can exclude the interfering factors such as base effect and weight fluctuation.

However, there are some limitations in this study. First, to maximally rule out the placebo effect and subjective bias, a standardized acupoint regimen is applied in this trial, which is not in accordance with the concept of individualized therapy of CTM. And additional studies are needed to determine the difference between the standardized acupoint regimen and the individualized acupoint regime. Second, according to TCM, de qi, the needle sensation during acupuncture, is regarded as an important sign of successful acupuncture, and is essential for exerting the clinical efficacy of acupuncture. However, for blinding purpose, acupuncturist are forbidden to enquire about it in both groups in this trial. Finally, as acupuncture is common approach in dealing various symptoms in China, chances are good that some participants in the sham-acupuncture group may feel the difference. Double blindness is not applied in this study, given that the procedure is conducted by the acupuncturists.

In conclusion, this article presents a rigorously designed trial to verify the efficacy of acupuncture on BCRL. The results of this trial will provide more information on this issue.

\section{Additional file}

\author{
Additional file 1: Block Randomized Sequence. (PDF $83 \mathrm{~kb}$ ) \\ Additional file 2: Sample Size Calculation and G Power Manual. (PDF 65 kb)
}

\begin{abstract}
Abbreviations
ARLVR: Absolute reduced limb volume ratio; BCRL: Breast cancer related lymphedema; Cl: Confidential interval; DSMB: Data and Safety Monitoring Board; ECG: Electrocardiograph; ES: effect size; GPH: Global Physical Health; GMH: Global Mental Health.; IAE: Incidence of adverse events; ITT: Intent-totreat; PP: per-protocol; PROMIS: Patient-Reported Outcomes Measurement Information System; SD: Standard deviation; TCC: Trial coordinating committee; TCM: Traditional Chinese medicine; TSC: Trial steering committee
\end{abstract}

\section{Acknowledgements}

We are grateful to Dr. Beverley de Valois (Lynda Jackson Macmillan Centre, UK) for her advice and guidance.

\section{Funding}

This study is funded by Key Laboratory Construction of Tumor Diseases Prevention in Liu Zhou, Guang Xi (No.2014G020403), China. Funder will have no role in the trial design, the manuscript writing, or the decision making for publication.

\section{Availability of data and materials}

The datasets used and/or analysed during the current study available from the corresponding author on reasonable request.

\section{Authors' contributions}

$\mathrm{SD}$ is the chief designer of this trial. ZP and $J \mathrm{~L}$ drafted the protocol. $\mathrm{HZ}$ and $\mathrm{XL}$ provided clinical advice. $J \mathrm{~L}, \mathrm{HZ}$ and $\mathrm{XL}$ are the coordinators and responsible for the screening and enrollment of patients. MD, YZ, LS, JC, BH, $F Q, H L, C W$, and $G Z$ are involved in the recruitment of patients. $J L$ and $X L$ drew pictures. SD, ZP and $\mathrm{YH}$ revised the manuscript. All authors have read and approved the final version of the manuscript.

\section{Ethics approval and consent to participate}

The trial was approved by the Institutional Review Board of the Fourth Affiliated Hospital of Guangxi Medical University (Approval Number: PJK2016090). All participants will provided written, signed informed consent. 


\section{Consent for publication}

Not applicable.

\section{Competing interests}

The authors declare that they have no competing interests.

\section{Publisher's Note}

Springer Nature remains neutral with regard to jurisdictional claims in published maps and institutional affiliations.

\section{Author details}

'Department of Galactophore, the Third Affiliated Hospital of Guangxi University of Chinese Medicine, Liuzhou, Guangxi, China. ${ }^{2}$ Department of Clinical Laboratory, the Fourth Affiliated Hospital of Guangxi Medical University, Liuzhou, Guangxi, China. ${ }^{3}$ Department of Clinical Laboratory, Liuzhou Maternity and Child Health Care Hospital, Liuzhou, Guangxi, China.

Received: 7 July 2017 Accepted: 19 September 2017

Published online: 21 September 2017

\section{References}

1. Kissin MW, Querci della Rovere G, Easton D, Westbury G. Risk of lymphoedema following the treatment of breast cancer. Br J Surg. 1986;73(7):580-4.

2. Paskett ED, Dean JA, Oliveri JM, et al. Cancer-related lymphedema risk factors, diagnosis, treatment, and impact: a review. J Clin Oncol. 2012;30(30):3726-33.

3. Petrek JA, Senie RT, Peters $M$, et al. Lymphedema in a cohort of breast carcinoma survivors 20 years after diagnosis. Cancer. 2001;92(6):1368-77.

4. DiSipio T, Rye S, Newman B, et al. Incidence of unilateral arm lymphoedema after breast cancer: a systematic review and meta-analysis. The Lancet Oncology. 2013;14(6):500-15.

5. Armer JM, Stewart BR. Post-breast cancer lymphedema: incidence increases from 12 to 30 to 60 months. Lymphology. 2010;43(3):118-27.

6. Chen W, Zheng R, Baade PD, et al. Cancer statistics in China, 2015[J]. CA Cancer J Clin. 2016;66(2):115-32.

7. Hayes SC, Johansson K, Stout NL, et al. Upper-body morbidity after breast cancer: incidence and evidence for evaluation, prevention, and management within a prospective surveillance model of care. Cancer. 2012:118(8 Suppl):2237-49.

8. Shih YC, Xu Y, Cormier JN, et al. Incidence, treatment costs, and complications of lymphedema after breast cancer among women of working age: a 2-year follow-up study. J Clin Oncol. 2009;27(12):2007-14.

9. Stout NL, Pfalzer LA, Springer B, et al. Breast cancer-related lymphedema: comparing direct costs of a prospective surveillance model and a traditional model of care. Phys Ther. 2012;92(1):152-63.

10. Meeske KA, Sullivan-Halley J, Smith AW, et al. Risk factors for arm lymphedema following breast cancer diagnosis in Black women and White women. Breast Cancer Res Treat. 2009;113(2):383-91.

11. Kilbreath SL, Refshauge KM, Beith JM, et al. Risk factors for lymphoedema in women with breast cancer: A large prospective cohort, vol. 28. Edinburgh, Scotland: Breast; 2016. p. 29-36.

12. Mori T, Lustman A, Katz-Leurer M. Self-measurement of upper extremity volume in women post-breast cancer: reliability and validity study. Physiotherapy theory and practice. 2015;31(4):283-7.

13. Fu MR, Rosedale M. Breast cancer survivors' experiences of lymphedemarelated symptoms. J Pain Symptom Manag. 2009;38(6):849-59.

14. Fu MR, Kang Y. Psychosocial impact of living with cancer-related lymphedema. Semin Oncol Nurs. 2013;29(1):50-60.

15. Chachaj A, Malyszczak K, Pyszel K, et al. Physical and psychological impairments of women with upper limb lymphedema following breast cancer treatment. Psycho-Oncology. 2010;19(3):299-305.

16. Taghian NR, Miller CL, Jammallo LS, et al. Lymphedema following breast cancer treatment and impact on quality of life: a review. Crit Rev Oncol Hematol. 2014;92(3):227-34.

17. Reyes-Gibby CC, Anderson KO, Morrow PK, et al. Depressive symptoms and health-related quality of life in breast cancer survivors. Journal of women's health (2002). 2012;21(3):311-8

18. Pinto M, Gimigliano F, Tatangelo F, et al. Upper limb function and quality of life in breast cancer related lymphedema: a cross-sectional study. European journal of physical and rehabilitation medicine. 2013:49(5):665-73.

19. Park JE, Jang HJ, Seo KS. Quality of life, upper extremity function and the effect of lymphedema treatment in breast cancer related lymphedema patients. Annals of rehabilitation medicine. 2012;36(2):240-7.
20. McNeely ML, Magee DJ, Lees AW, Bagnall KM, Haykowsky M, Hanson J. The addition of manual lymph drainage to compression therapy for breast cancer related lymphedema: a randomized controlled trial. Breast Cancer Res Treat. 2004:86(2):95-106.

21. Ezzo J, Manheimer E, McNeely ML, et al. Manual lymphatic drainage for lymphedema following breast cancer treatment. Cochrane Database Syst Rev. 2015;5

22. Penha T, IJsbrandy C, Hendrix N, et al. Microsurgical techniques for the treatment of breast cancer-related lymphedema: a systematic review. J Reconstr Microsurg. 2013;29(2):99-106.

23. Omar MTA, Shaheen AAM, Zafar H. A systematic review of the effect of lowlevel laser therapy in the management of breast cancer-related lymphedema. Support Care Cancer. 2012;20(11):2977-84.

24. Tervala TV, Hartiala P, Tammela T, et al. Growth factor therapy and lymph node graft for lymphedema. J Surg Res. 2015;196(1):200-7.

25. Stuiver MM, ten Tusscher MR, Agasi-Idenburg CS, et al. Conservative interventions for preventing clinically detectable upper-limb lymphoedema in patients who are at risk of developing lymphoedema after breast cancer therapy. Cochrane Database Syst Rev. 2015;2:Cd009765.

26. Cheifetz $\mathrm{O}$, Haley L. Management of secondary lymphedema related to breast cancer. Can Fam Physician. 2010;56(12):1277-84.

27. Jiang M, Zhang $C$, Zheng $G$, et al. Traditional Chinese medicine Zheng in the era of evidence-based medicine: a literature analysis. Evid Based Complement Alternat Med. 2012;2012

28. Kaptchuk TJ. Acupuncture: theory, efficacy, and practice. Ann Intern Med. 2002;136(5):374-83.

29. White A, Ernst E. A brief history of acupuncture. Rheumatology. 2004;43(5):662-3.

30. Cassileth BR, Van Zee KJ, Chan Y, et al. A safety and efficacy pilot study of acupuncture for the treatment of chronic lymphoedema. Acupunct Med. 2011:29(3):170-2.

31. Cassileth BR, Van Zee KJ, Yeung KS, et al. Acupuncture in the treatment of upper-limb lymphedema: results of a pilot study. Cancer. 2013;119(13):2455-61.

32. Jeong YJ, Kwon HJ, Park YS, et al. Treatment of Lymphedema with Saam Acupuncture in Patients with Breast Cancer: A Pilot Study. Medical acupuncture. 2015;27(3):206-15.

33. de Valois BA, Young TE, Melsome E. Assessing the feasibility of using acupuncture and moxibustion to improve quality of life for cancer survivors with upper body lymphoedema. Eur J Oncol Nurs. 2012;16(3):301-9.

34. Smith CA, Pirotta M, Kilbreath S. A feasibility study to examine the role of acupuncture to reduce symptoms of lymphoedema after breast cancer: a randomised controlled trial. Acupuncture in Medicine 2014:acupmed2014-010593.

35. Yao $C, X u Y, C$ hen $L$, et al. Effects of warm acupuncture on breast cancerrelated chronic lymphedema: a randomized controlled trial. Current oncology (Toronto, Ont). 2016;23(1):e27-34.

36. Hui KK, Nixon EE, Vangel MG, et al. Characterization of the" deqi" response in acupuncture. BMC complementary and alternative medicine 2007;7(1):1. 1

37. Park JE, Ryu YH, Liu Y, Jung HJ, Kim AR, Jung SY, Choi SM. A literature review of de qi in clinical studies. Acupunct Med. 2013;31(2):132-42.

38. Streitberger K, Kleinhenz J. Introducing a placebo needle into acupuncture research. Lancet. 1998;352(9125):364-5.

39. Andersen L, Højris I, Erlandsen M, et al. Treatment of breast-cancer-related lymphedema with or without manual lymphatic drainage: a randomized study. Acta Oncol. 2000;39(3):399-405.

40. Hays RD, Bjorner JB, Revicki DA, Spritzer KL, Cella D. Development of physical and mental health summary scores from the patient-reported outcomes measurement information system (PROMIS) global items. Qual Life Res. 2009;18(7):873-80.

41. Cassileth BR, Van Zee KJ, Yeung KS, et al. Acupuncture in the treatment of upper-limb lymphedema[J]. Cancer. 2013;119(13):2455-61.

42. Dominick SA, Madlensky L, Natarajan L, et al. Risk factors associated with breast cancer-related lymphedema in the WHEL Study. Journal of cancer survivorship: research and practice. 2013;7(1):115-23.

43. McLaughlin SA, Bagaria S, Gibson T, et al. Trends in risk reduction practices for the prevention of lymphedema in the first 12 months after breast cancer surgery. J Am Coll Surg. 2013;216(3):380-9.

44. Greenslade MV, House CJ. Living with lymphedema: a qualitative study of women's perspectives on prevention and management following breast cancer-related treatment. Canadian Oncology Nursing Journal/Revue canadienne de soins infirmiers en oncologie. 2006;16(3):165-71.

45. White $P$, Lewith $G$, Hopwood $V$, Prescott $P$. The placebo needle, is it a valid and convincing placebo for use in acupuncture trials? A randomised, singleblind, cross-over pilot trial. Pain. 2003;106(3):401-9. 
46. McManus CA, Schnyer RN, Kong J, et al. Sham acupuncture devices-practical advice for researchers. Acupunct Med. 2007;25(1-2):36-40.

47. Schnyer RN, Iuliano D, Kay J, et al. Development of protocols for randomized sham-controlled trials of complex treatment interventions: Japanese acupuncture for endometriosis-related pelvic pain. The Journal of Alternative and Complementary Medicine. 2008;14(5):515-22.

48. Adriaenssens N, Buyl R, Lievens P, et al. Comparative study between mobile infrared optoelectronic volumetry with a Perometer and two commonly used methods for the evaluation of arm volume in patients with breast cancer related lymphedema of the arm. Lymphology. 2013;46(3):132-43.

49. Lee MJ, Boland RA, Czerniec S, et al. Reliability and concurrent validity of the perometer for measuring hand volume in women with and without lymphedema. Lymphat Res Biol. 2011;9(1):13-8.

50. Bulley C, Coutts F, Tan CW. Perometry limb volume measurement: protocol development and reliability. The European Journal of Physiotherapy. 2013; 15(4):193-200

51. Tan CW, Coutts F, Bulley C. Measurement of lower limb volume: agreement between the vertically oriented perometer and a tape measure method. Physiotherapy. 2013;99(3):247-51.

52. Engelberger RP, Blazek C, Amsler F, et al. Reproducibility and day time bias correction of optoelectronic leg volumetry: a prospective cohort study. BMC Med Res Methodol. 2011:11:138.

\section{Submit your next manuscript to BioMed Central} and we will help you at every step:

- We accept pre-submission inquiries

- Our selector tool helps you to find the most relevant journal

- We provide round the clock customer support

- Convenient online submission

- Thorough peer review

- Inclusion in PubMed and all major indexing services

- Maximum visibility for your research

Submit your manuscript at www.biomedcentral.com/submit 